\title{
FAUSTINO BALLVÉ PALLISÉ Y SUS DIEZ LECCIONES DE ECONOMÍA (1956)
}

\author{
MANUEL MARTÍN RODRÍGUEZ*
}

Resumen: Faustino Ballvé Pallisé (1887-1958), como casi todos lo economistas del exilio republicano español de 1939, sigue siendo un gran desconocido en su propio país, pese a sus influyentes Diez Lecciones de Economía (1965), publicadas en varias idiomas y en numerosas ediciones, y actualmente un clásico de la escuela austriaca de economía. En este trabajo se ofrece una amplia semblanza de este economista catalán, personal e intelectualmente vinculado a Mises, y se analizan las fuentes, la estructura, el contenido y la recepción internacional de sus Lecciones.

Palabras clave: Faustino Ballvé; escuela austriaca de economía; exilio republicano español.

\section{Clasificación JEL: B31.}

Abstract: Faustino Ballvé Pallisé (1887-1958), like almost all the economists of Spanish republican exile of 1939, continues being a great unknown in his own country, in spite of his influential Ten Lessons of Economics, published in several languages and in numerous editions, and at present a classic

* Manuel Martín Rodríguez es académico de número de la Academia de Ciencias Sociales y del Medio Ambiente de Andalucía y correspondiente de la Real Academia de Ciencias Morales y Políticas. A lo largo de su dilatada carrera académica ha sido catedrático de Economía Aplicada de la Universidad de Granada, director de la Revista de Estudios Regionales y miembro del consejo editorial de la Revista de Historia Económica y de la Revista de Economía Aplicada. Autor de numerosas publicaciones sobre historia del análisis y del pensamiento económico en España, entre sus trabajos en este campo destacan sus libros Pensamiento económico español sobre la población (1986), Cinco grandes economistas andaluces ante el comercio internacional (1994), Análisis económico y revolución liberal en España (2009), sus varias colaboraciones en E. Fuentes Quintana (dir.), Economía y economistas españoles (1999-2004), y sus importantes estudios introductorios a Vicente Montano, Arcano de Príncipes (1986), Valle Santoro, Elementos de Economía Política, con aplicación práctica a España (1989) y Carlos de Orduña Zarauz, Lecciones de Economía matemática (2006).

Procesos de Mercado: Revista Europea de Economía Política Vol. VII, n. ㄹ 2, Otoño 2010, pp. 13 a 36 
work of the Austrian School of economics. This article offers a short biography of this Catalan economist, personally and intellectually linked to Mises, and analyses the sources, the structure, the contents and the internacional reception of his Lessons.

Key words: Faustino Ballvé; Austrian School of Economics; Spanish Republican Exile.

JEL Classification: B31.

LA VIDA DE BALLVÉ ANTES DE SU EXILIO (1887-1939)

Faustino Ballvé Pallisé ${ }^{1}$ (Barcelona, 1887; México, 1958) se licenció en Derecho en la Universidad de Barcelona en 1907. En 1912, fue becado por la Junta de Ampliación de Estudios para estudiar en Berlín con el jurista Franz von Liszt. Su objetivo entonces era el de opositar a una cátedra de Derecho Penal, para lo que durante su estancia en Alemania preparó un importante trabajo, que tendría una gran repercusión en España en los años siguientes y, más tarde, en distintos países latinoamericanos: $\mathrm{La}$ teoría jurídica del delito según Beling. ${ }^{2}$ Sin embargo, su fracaso en unas primeras oposiciones que se celebraron en 1913, en las que el tribunal no entendió sus novedosos puntos de vista, le hizo abandonar prematuramente su carrera académica. ${ }^{3}$

Después de esta experiencia, probablemente influido por Manuel Raventós, también becario de la Junta para la Ampliación de Estudios, con quien había coincidido en Berlín, decidió irse a estudiar economía a Londres, donde se ganó la vida con

\footnotetext{
${ }^{1}$ En algunos textos, en lugar de Pallisé, aparece como segundo apellido el de Pellicer. En los diversos escritos firmados por él mismo que he podido conocer, siempre figura Ballvé Pallisé, o simplemente Ballvé.

2 El trabajo fue publicado en la editorial Fortanet (Barcelona, 1913) y en Anales de la Junta para la Ampliación de Estudios e Investigaciones Científicas, tomo IX (Madrid, 1913). En este mismo tomo, se publicó otro trabajo suyo, también sobre derecho penal, «Las últimas investigaciones estadísticas de la criminalidad en Alemania».

3 Vid. Pere Bosch i Gimpera (1980), Memóires, 15; y Francisco Javier Dosil Mancilla, «La JAE peregrina» (Revista de Indias, 2007, LXVII, n. 239: 307-332).
} 
traducciones del alemán y del inglés. De estos años son sus traducciones de A. Eleutheropulos (Sociología, Madrid: Reus, 1913), Ludwig Gurlitt (La educación natural, Madrid: La Lectura, 1914), Chapman S.J. (Economía Política, Barcelona: Ibérica, 1915, en colaboración con Manuel Raventós), H. Herckner (La cuestión obrera, Madrid: Reus, 1916), A. Damaschke (La reforma agraria, Madrid: Reus, 1916), Otto Hintze et al. sobre Alemania y la Guerra Europea (1916) ${ }^{4}$ y de la novela de A.E.W. Mason, El misterio de la Villa Rosa, publicada en París en 1913. Además de todo ello, en Londres entró en contacto con el diplomático y poeta colombiano Santiago Pérez Triana (Bogotá, 1858; Londres, 1916), con quien colaboró, junto con otros españoles, entre ellos Araquistain, en su revista Hispania.

A su vuelta a España, abrió un bufete especializado en asuntos mercantiles e internacionales, con gran éxito, llegando a ser miembro de la Academia de Jurisprudencia y Legislación, de la Sociedad de Estudios Económicos de Barcelona, de la International Law Association y de la Sociedad de Derecho Comparado de Berlín. En sus primeros años de ejercicio de la abogacía, continuó interesándose por el socialismo que había estudiado en Londres, se afilió a la Agrupación Socialista de Barcelona y publicó el libro El socialismo y la guerra (Barcelona: Estudio, 1916), en el que se ocupó de la Internacional en la Primera Guerra Mundial, dejándose notar su inicial formación germanófila.

Decepcionado por el socialismo, abrazó el republicanismo liberal, llegando a dirigir en Barcelona el partido Izquierda Republicana, de Azaña, por el que resultó elegido diputado en las elecciones del 16 de febrero de 1936, en las que obtuvo 262.670 votos por la circunscripción de Barcelona capital, a muy poca distancia de Nicolau d'Olwer el político catalán más votado en esas elecciones. En esta etapa de su vida, aunque dedicó mucho tiempo a la política, continuó con su despacho profesional y con su trabajo de traductor y ensayista. Tradujo a W. Lexis (El comercio,

4 Otto Hintze, Fiedrich Meinecke, Hermann Oucken y Hermann Schumacher, Alemania y la Guerra Europea, vol. 1; Fiedrich Tezner, Aliados y enemigos de Alemania, vol. 2; y Hermann Oucken, Génesis y espíritu de la Guerra, vol. 3. Los tres volúmenes fueron publicados por la editorial Gustavo Gili, en 1916. 
1929) y T. Niemeyer (Derecho Internacional Público, Barcelona: Labor, 1930), y publicó Spanien als betätigunsfeld für fremden andel und industrie (Essen: Baedeker, 1926), un interesante texto sin traducir aun al español.

\section{II \\ LOS AÑOS DE EXILIO EN MÉXICO (1941-1958)}

\section{La enseñanza de la Economía en México en 1939}

Para entender las Diez Lecciones de Economía (1956b) de Ballvé y su propia trayectoria académica en México, a donde se vio obligado a exiliarse al terminar la guerra civil tras una breve estancia en Francia, resulta indispensable un breve apunte previo sobre la situación de los estudios de Economía en este país al tiempo de la llegada de los republicanos españoles en 1939.

El único centro universitario en el que se impartían estudios de economía, la Escuela Nacional de Economía de la Universidad Nacional Autónoma de México (UNAM), tenía sus orígenes en 1929, fecha en que, formando parte de un plan general para que el país pudiera disponer de economistas que contribuyeran eficazmente a su desarrollo económico, ${ }^{5}$ Daniel Cosío, junto a

\footnotetext{
5 Durante los años veinte y treinta, se habían creado en México un conjunto de nuevas instituciones económicas orientadas al desarrollo económico del país: el Banco de México (1925), un verdadero banco central, aunque todavía sin algunas de las principales funciones de este tipo de bancos; la Nacional Financiera, un banco de desarrollo industrial; el Banco de Crédito Agrícola (1926), relacionado con la reforma agraria; y el Departamento de Estadística Nacional (1924), que debía proporcionar la información necesaria para los programas de desarrollo. Al mismo tiempo, en la Secretaría de Hacienda, Jesús Silva Herzog había emprendido la tarea de organizar una biblioteca especializada en economía y había creado en 1928 el Instituto Mexicano de Investigaciones Económicas, para estudiar la economía mexicana «sin necesidad de importar sistemas extraños». Un excelente análisis de la estrecha relación entre los objetivos económicos del Estado y la institucionalización de los estudios de economía en México, en Babb (2001): Managing México. Economists from Nationalism to Neoliberalism, Princeton: Princeton University Press; y (2005): «Del nacionalismo al neoliberalismo: El acenso de los nuevos Money Doctors en México», en Daniel Mato (coord.), Políticas de economía, ambiente y sociedad en tiempos de globalización, Caracas: Facultad de Ciencias Económicas y Sociales, Universidad Central de Venezuela, 2005: 155-172.
} 
otros intelectuales mexicanos que, como él, habían estudiado economía en el extranjero, ${ }^{6}$ había conseguido que se creara una Sección de Economía dentro de la Facultad de Derecho y Ciencias Sociales. Los primeros profesores de la Escuela estuvieron muy influidos por el marxismo y otras ideologías socialistas, pero siempre de forma un tanto confusa ya que jamás trataron de promover una verdadera revolución social y política, sirviéndose simplemente de estas ideas para justificar la creciente intervención del Estado en la vida económica.

Jesús Silva Herzog, nombrado director de la Escuela para el periodo 1940-42, acometió importantes reformas y modificó su plan de estudios. El minucioso análisis de las tesis doctorales presentadas, realizado recientemente por Babb (2002: 48-66), ha puesto de manifiesto que, aun manteniendo su inicial orientación izquierdista, el centro fue adaptándose a los cambios que iban teniendo lugar en el país. Si en el periodo de Cárdenas (1934-40) la preocupación fundamental había sido la reforma agraria, en los años siguientes, los del milagro económico mexicano (1940-70), el mayor interés se centró en los procesos de crecimiento económico y de industrialización promovidos por el gobierno. Por otra parte, la confusión inicial, que había identificado marxismo con intervención económica del Estado, se fue disipando por la introducción del keynesianismo y de las ideas económicas de la Comisión Económica para América Latina (CEPAL).

El segundo centro superior en impartir estudios de economía, ya con los republicanos españoles en México, fue La Casa de Espa$\tilde{n} a-E l$ Colegio de México, cuya historia es bien conocida. ${ }^{7} \mathrm{Al}$ poco

6 Entre éstos, estuvieron Villaseñor, Palacios y Espinosa de los Monteros. Eduardo Villaseñor había aprovechado su estancia en Londres como agregado comercial de la embajada de México para estudiar en la London School of Economics. Miguel Palacios Macedo estuvo exiliado en París durante cinco años, que había ocupado en el estudio de la economía. Antonio Espinosa de los Monteros había hecho el bachillerato en un colegio norteamericano y luego había cursado un master de economía en Harvard, donde había coincidido con Cosío (Memorias, México, D.F.: Editorial Joaquín Mortiz, S.A., 1976: 139).

7 La Casa de España y, a partir de septiembre de 1940, su sucesora, El Colegio de México, estuvieron presididos por Alfonso Reyes hasta 1959, en que le sucedió Cosío, 
de su fundación, en 1943, el sociólogo español Medina Echavarría fue encargado de dirigir el Centro de Estudios Sociales, creado en su seno también con la finalidad de «dotar al gobierno mexicano de cuadros intelectuales bien preparados». ${ }^{8}$ Mientras funcionó, su plan de estudios estuvo organizado en cursos semestrales con una duración total de cuatro años. De la enseñanza de la economía se ocupó el economista mexicano Victor L. Urquidi, pero los españoles Javier Márquez, Herrero y Sánchez Sarto, impartieron cursos de economía ocasionales. ${ }^{9}$ La orientación de sus enseñanzas fue weberiana en sociología y keynesiana en economía, y, contrariamente a lo que venía ocurriendo en la Escuela Nacional de Economía, el gran ausente de sus aulas fue Marx. En 1960, Cosío, nuevo director, acometió su modernización, consiguiendo que dos años más tarde el presidente López Mateos firmara un decreto por el que se le reconocía el carácter de «escuela de tipo universitario» con la posibilidad de impartir cualquier tipo de conocimientos.

La deriva izquierdista de la Escuela Nacional de Economía, preocupaba a empresarios y alumnos, por las dificultades que este tipo de enseñanzas representaba para el reclutamiento de buenos profesionales al servicio de la empresa. Por ello, algunos grandes bancos y empresarios mexicanos pensaron en promover otras ofertas educativas más orientadas al mercado. En 1943, un grupo de industriales fundó el Instituto Tecnológico de Monterrey (ITESM), inspirado en los de Massachusetts y California, en principio sólo para formar a ingenieros y licenciados en administración de empresas, pero que dispuso ya de un departamento de economía en 1954. ${ }^{10}$ Y en 1946, el Banco de México y otros grandes bancos y empresas mexicanas fundaron la Asociación Cultural Mexicana,

que había sido su secretario durante los primeros años (vid. Lida y Matesanz, 1988: La Casa de España en México, México: El Colegio de México; y Lida et al., 2000: La Casa de España y El Colegio de México. Memoria 1938-2000, México: El Colegio de México).

8 Lida et al. (2000): 236.

9 El Centro de Estudios Sociales de El Colegio de México cerró en 1946. Sobre su primera y única promoción, vid. González Navarro (2003): «José Medina Echavarría», Letras Libres, 1 de agosto de 2003.

10 D.C. Levy (1980): University and Government in México: Autonomy in an Authoritarian System, New York: Praeger. 
con el objetivo principal de crear el Instituto Tecnológico Méxicano (ITM, luego ITAM), cuyo primer programa docente fue el de economía, al que se unieron pronto algunos «disidentes» de la Escuela Nacional de Economía, como el propio Ballvé, quien para entonces ya había conocido a Mises y abandonado completamente sus primeras veleidades socialistas.

Por otra parte, la evolución de la economía mexicana en estos años puede resumirse también en pocas líneas. Después de la nacionalización de las industrias básicas y de las grandes reformas sociales realizadas por Cárdenas (1934-40), México entró, bajo la presidencia de Ávila Camacho (1940-46), en una etapa más tranquila en la que el desarrollo económico del país fue prioritario, atribuyéndose un papel central al sector público, que puso en práctica una arriesgada política inflacionista, con tipos de cambio fijos y un sistema fiscal insuficiente, que conduciría finalmente a un fuerte déficit de la balanza de pagos, a un primer ajuste monetario en 1948, con el apoyo del FMI y del Banco Mundial, y a una devaluación en 1954, de 8,65 a 12,5 pesos por dólar. ${ }^{11}$

A estos centros superiores de enseñanza de la economía, y en esta situación política y económica, fue a donde se incorporaron los exiliados españoles en México. Aunque fueron más, citemos aquí como los más destacados de quienes lo hicieron a Javier Márquez Blasco, Ramón Ramírez Gómez, Alfredo Lagunilla Iñarritu, Sánchez Sarto, Antonio Sacristán Colás y el propio Faustino Ballvé. ${ }^{12}$

11 R.D. Hansen (1971): The Politics of Mexican Development, Baltimore: The Johns Hopkins University Press; y E. Cárdenas (1956): La política económica en México, 19501994, México: FCE.

12 Un estudio general sobre los economistas españoles del exilio de 1939, en M. Martín Rodríguez y E. Fernández Clemente (2009): «Sesenta economistas académicos del exilio (1936-1939)», en E. Fuentes Quintana (dir.) y F. Comín Comín (coord.): Economía y economistas españoles en la guerra civil, 2 vols., Madrid: Real Academia de Ciencia Morales y Políticas y Galaxia Gutenberg Círculo de Lectores. 


\section{La estancia de Ballvé en México}

Ballvé llegó a México en 1941. Después de convalidar su título de abogado, abrió de nuevo su despacho profesional (Bufete Ballvé \& Murat), ${ }^{13}$ continuó con sus traducciones ${ }^{14}$ y se dedicó a la docencia del derecho y de la economía, primero en la Universidad Nacional Autónoma de México (UNAM) y, a partir de 1948, también en el Instituto Tecnológico de México (ITAM).

Recién llegado a México, conoció a Mises, que había sido invitado por Montes de $\mathrm{Oca}^{15}$ a impartir unas conferencias en la ciudad de México durante los meses de enero y febrero de 1942. ${ }^{16}$ En ellas, el economista austriaco presentó una economía muy distinta a la que se enseñaba entonces en la Escuela Nacional de Economía. Criticó el modelo de «puertas cerradas para la industrialización», la política inflacionista y la transigencia con las reivindicaciones sindicales de elevación de salarios que se venían practicando en México. Sostuvo que la única vía para que la economía mexicana pudiera competir en los mercados internacionales era la de continuar con producciones intensivas en

${ }^{13}$ Ballvé dio numerosas conferencias sobre Derecho, reeditó algunos de sus trabajos publicados en España y publicó otros nuevos, siempre relacionados con el delito: Función de la tipicidad en la dogmática del delito, México: UNAM, 1951; y El problema de la participación en el delito, Mexico: Facultad de Derecho, UNAM, 1954.

${ }^{14}$ Sus traducciones de esta época fueron: S.E. Morison, Historia de los Estados Unidos de Norteamérica, 1951 (en colaboración con Odón Durán d’Ocón); y R. Stammler, Modernas teorías del Derecho y del Estado, México: Ediciones Botas, 1955.

15 Luis Montes de Oca, que había participado en la revolución mexicana, había sido nombrado director del Banco de México en 1935, cargo en el que permaneció durante buena parte de la presidencia de Cárdenas, moderando en este tiempo sus primitivas posiciones. Al dejarlo en 1940, fundó el Banco Internacional y comenzó a distinguirse por sus ideas liberales. En 1961, tradujo al español El Socialismo, de Mises (México: Hermes).

16 Año y medio después, en junio de 1943, Mises volvió a ser invitado por una asociación de empresarios mexicanos a elaborar un informe sobre la economía de México, inédito hasta su publicación muy recientemente. La versión original inglesa ha aparecido en un volumen titulado Selected Essays of Ludwig von Mises: The Political Economy of International Economic Reform and Reconstruction (pp. 203-253), publicado por el Liberty Fund Inc. de Indianápolis en 1999, en el que se recogieron textos inéditos de Mises escritos a principios de los años cuarenta. Y poco antes, había sido publicado en español por el Instituto Cultural Ludwig von Mises de México, con el título de Problemas económicos de México: Ayer y hoy (México, 1998). 
mano de obra, aumentar la formación de capital y alentar procesos migratorios hacia países con mayor productividad. Y recomendó un reforzamiento de los derechos de propiedad, en una dirección absolutamente contraria a la que se había seguido desde el comienzo de la revolución.

El mensaje de las conferencias de Mises no era fácilmente asimilable en el México de ese tiempo, pero entusiasmó a Ballvé, que había ido abdicando de su primer socialismo. A partir de entonces, facilitada por el idioma, se inició entre ambos una amistad personal e intelectual, que duraría ya hasta la muerte del economista español en octubre de 1958. Además de con una extensa correspondencia, ${ }^{17}$ Ballvé cultivó esta relación acompañando a Mises y a sus amigos de la NYU en algunas de sus iniciativas y adoptando él mismo otras similares en México, a algunas de las cuales nos referimos a continuación.

Aunque no figuró en la lista de los 39 académicos que en 1947 participaron en el primer meeting de la Mont Pelèrin Society, su vinculación a esta organización internacional liberal debió de ser casi inmediata. ${ }^{18}$ Aparte de que lo pudiera haber hecho en otros anteriores, sabemos que en el octavo meeting, que tuvo lugar en Saint Moritz (Suiza) en septiembre de 1957, presentó una breve ponencia, titulada «The Meaning of Liberty and the Philosophical Basis of Liberalism», en un panel en el que participaron también W.H. Chamberlin, F.A. Harper, L. Rougier y F.A. Hayek. ${ }^{19}$

En 1955, M. Sennholz ${ }^{20}$ le solicitó un trabajo para incluir en un libro homenaje conmemorativo del 50 aniversario del doctorado

17 Los papeles de Mises se encuentra actualmente en los archivos del Grove City College (Pensilvania, USA), en el que enseñaron economía dos de sus más cercanos alumnos de la NYU, Hans F. Sennholz e Israel Kirzner. La correspondencia entre Mises y Ballvé, en Grove City Archive: Ballvé files.

18 Entre los asistentes a esta primera conferencia, estuvieron, entre otros, Eucken, Milton Friedman, Hazlitt, Knight, Popper, Mises, Robbins, Röpke, Stigler y el español Salvador de Madariaga (htpp: / / www.montpelerin.org).

19 Mont Pèlerin Society: Inventory of the General Meeting Files (1947-1998). Preface by Jacques van Offelen, Ghent (Belgium): Liberaal Archief

20 Los profesores Hans F. Sennholz y su esposa M. Sennholz tuvieron relaciones verdaderamente familiares con el matrimonio Mises durante su estancia en la NYU. Les acompañaron en sus viajes académicos al extranjero, les visitaron con frecuencia en su casa neoyorkina, e incluso aceptaron que Margit Mises fuera la madrina de su hijo. 
de Mises en la Universidad de Viena, que se publicó el año siguiente con el título de On Freedom and Free Enterprise (1956a), incluyendo ensayos de amigos y seguidores de Mises, entre ellos, Röpke, Hayek, Rappard, Jouvenal, Hazlitt, Rothbard y Rueff. El trabajo remitido por Ballvé fue «On Methodology in Economics», al que nos referiremos más extensamente en el próximo epígrafe. ${ }^{21}$

Desde el Ateneo Libertad de México D.F., del que fue presidente, Ballvé se convirtió en un verdadero propagandista de la economía liberal, organizando actos, dictando conferencias, participando en programas de radio y escribiendo textos breves, que alcanzaron una notable difusión, no sólo en México sino también en otros países latinoamericanos, particularmente en Guatemala y Perú. Parte de estos textos fueron publicados en periódicos y tiradas especiales y algunos de ellos han sido reiteradamente reproducidos en boletines de distintos centros liberales. Basten aquí dos únicas referencias a toda esta actividad. ${ }^{22}$

En una conferencia pronunciada en el Ateneo Libertad en 1954, con el título de Libertad y Economía, ${ }^{23}$ Ballvé arremetió contra W.A. Lewis, un economista nacido en la isla de Santa Lucía que había estudiado en la London School of Economics y trabajado luego al servicio de organizaciones internacionales en programas de desarrollo económico de países del Caribe, que con su

M. Sennholz invitó a participar en el libro exclusivamente a «the most famous Mises friends and colleages», entre los que estuvieron Baudin, Rueff, Bertrand de Jouvenel, Hayek, Hazlitt, Machlup, Röpke, Rothbard, Hans Sennholz y el propio Faustino Ballvé.

${ }^{21}$ F. Ballvé (1956a): «On Methodology in Economics», en Mary Sennholz (ed.): On freedom and Free Enterprise. Essays in Honor of Ludwig von Mises, Princeton: D. Van Nostrand Company, Inc.. Años después, se ha publicado una versión en español de este texto en la Revista Libertas, V: 9 (octubre 1988), actualmente Revista de Instituciones, Ideas y Mercados (Revista RIIM), que publica ESEADE, un instituto de enseñanza superior e investigación fundado en Buenos Aires en 1978 por Alberto Benegas Linch, su primer rector, Federico Zorraquin y otros empresarios argentinos, con la participación de Hayek, Manuel F. Ayau (de la Universidad Francisco Marroquín de Guatemala) y otros intelectuales próximos a la escuela austriaca

22 Junto a los que se glosan en el texto a continuación, pueden citarse, entre otros: La crisis de la libertad, México: Ateneo Libertad, 1951; y La mixtificación de la democracia, México: Ateneo Libertad, 1951

23 «Libertad y Economía», Centro de Estudios Económico-Sociales (CEES) (Guatemala), año 2, febrero 1960, n.ํㅜ 6 . Este texto había sido publicado también por el Ateneo Libertad (1954). 
influyente libro, La planeación económica (1952), se había convertido en el principal soporte de la intervención estatal en los procesos de producción y distribución en los países latinoamericanos. Ballvé negó que estas políticas fueran eficaces, defendiendo la economía de mercado, la libertad de comercio exterior y la regulación automática del dinero. El nacionalismo económico, decía Ballvé en su refutación a las ideas de Lewis, no era la consecuencia de las guerras sino su causa, y el dirigismo económico, que atribuía al funcionamiento de la economía libre los ciclos económicos, los monopolios y el desempleo, no constituía más que una gran falacia.

Y en un texto sobre la Función social de la propiedad, ${ }^{24}$ analizó de nuevo la inutilidad de que el Estado interviniera para mejorar la producción y distribución de la riqueza, dando un nuevo paso en sus críticas. Lo más preocupante para Ballvé, era el «mito de la maldad de la propiedad privada, [...], tan deletéreo que ha llegado a afectar a los espíritus de muchos empresarios», haciendo que se avergüencen de la riqueza que tienen, cuando «en realidad no hacen otra cosa que administrar y fomentar el bien común, al paso que muchos de los que se ostentan como sus paladines, al predicar e imponer medidas utópicas, están dañando este bien común, aunque no siempre dañen sus propios intereses».

No he podido llegar a saber con certeza que Ballvé fuera ya masón en España, pero no hay duda de que perteneció a la Logia Libertad 233 del Valle de México, en la que el 14 de octubre de 1950, con motivo de su elevación al 20 grado, leyó un trabajo titulado Masonería y Libertad, en el que sostuvo estas mismas ideas. ${ }^{25}$

Por último, a estas actividades, hay que añadir que Ballvé nunca olvidó su interés por la política española, con una presencia continuada en actos y escritos políticos de republicanos exiliados. Por citar tan sólo uno de ellos, resulta especialmente significativo que el 11 de octubre de 1955, cuando ya se habían firmado los pactos entre Estados Unidos y España, pusiera su firma, como

\footnotetext{
24 «Función social de la propiedad», Centro de Estudios Económico-Sociales (CEES) (Guatemala), año 2, septiembre 1960, n. ${ }^{\circ} 13$.

25 F. Ballvé: Masonería y Libertad (1950), Centro Documental de la Memoria Histórica, Ministerio de Cultura (signatura F-11593, n.ํ de registro 34.626).
} 
presidente del Ateneo Libertad, junto a las de otros dirigentes de entidades del exilio para que la ONU rechazase la solicitud de la «España fascista, falangista y nacionalista» para ingresar en esta organización. ${ }^{26}$ Por esta dedicación, al cumplir los 70 años de edad, muy cerca ya de su muerte, el gobierno de la República en el exilio le concedería la orden de la liberación de España con el grado de Comendador. ${ }^{27}$

III

LAS DIEZ LECCIONES DE ECONOMÍA (1956B)

\section{Fuentes, estructura y contenidos}

Ballvé comenzó a dictar sus lecciones de Economía en la Facultad de Derecho de la UNAM, ya bajo la influencia de Mises. Aunque el medio no era el más adecuado, debió gozar de un cierto margen de libertad, alentado y protegido siempre por el Instituto de Investigaciones Sociales y Económicas, del que fue miembro de su Consejo Directivo. ${ }^{28}$

26 «Carta de los republicanos españoles al Secretario General de la ONU fundamentando y solicitando no se acepte a la España de Franco como miembro de la Organización» (1955). Además de por dirigentes de partidos políticos y sindicatos, la carta aparecía firmada, entre otros, por José Giral, de la Unión de Profesores Universitarios Españoles, y León Felipe, de la Unión de Intelectuales Españoles.

27 En un escrito con el membrete de International and Corporation Lawyers (Bufete Ballvé \& Murat), fechado de 5 de noviembre de 1957, Faustino Ballvé se dirigía al Congreso de la Unión, solicitando autorización para usar esta distinción en México. En su exposición, decía que «sin perjuicio de cumplir fielmente y con fervor mis deberes como mexicano, he seguido defendiendo la causa de la libertad de España y en general de todos los pueblos» (Diario de los Debates de la Cámara de Diputados del Congreso de los Estados Unidos Mexicanos, Legislatura XLIII, Año III, Periodo Comisión Permanente, fecha 1958-01-09, número de Diario 26).

${ }^{28}$ El Instituto de Investigaciones Sociales y Económicas A.C. de México D.F. había sido fundado por Gustavo R. Velasco y Agustín Navarro, ambos vinculados a la Mont Pèlerin Society. Velasco, sobrino de Montes de Oca, que había invitado a Mises a visitar México en 1941, fue directivo del Banco Internacional, fundado por el propio Montes de Oca; presidente de la Asociación de Banqueros de México (1950-51); presidente de la Confederación Patronal de la República de México (Coparmex), fundada para resistir los intentos de bolchevización de Mexico durante la presidencia de Álvaro Obregón (1920-24); y rector de la Escuela Libre de Derecho de México, fundada en 
A partir de su incorporación al ITAM en 1948, en el que impartió un curso de Economía, Ballvé se sintió mucho más libre y se consagró a la preparación de un texto que, como el de Mises (Human Action), pudiera ser entendido no sólo por sus alumnos sino «al menos por la parte culta y por los elementos directores de nuestra sociedad». Las versiones previas, que discutió con sus alumnos, y su correspondencia con Mises le sirvieron para fijar algunas de sus ideas y para encontrar el tono justo que él deseaba en su exposición. Y el resultado de todo ello fueron sus Diez Lecciones de Economía (1956b), un librito de 112 páginas en cuarto menor, cuya primera edición fue publicada por el Instituto de Investigaciones Sociales y Económicas A.C., de México D.F., con un breve prólogo de Gustavo R. Velasco.

Ballvé hizo explícitas sus fuentes en la lección I. De acuerdo con su análisis, la crisis de la escuela clásica se había debido a que sus pretendidas leyes económicas eran tan sólo simples regularidades, que fallaban a menudo en su aplicación, y a la creencia, «divulgada por la propaganda y aceptada irreflexiblemente por la intelectualidad y la clase media», de que la libre iniciativa tan sólo beneficiaba a unos pocos en contra de los intereses de los humildes y, en particular, de los trabajadores. Y en esta situación, Menger, partiendo de su teoría de la utilidad marginal, había hecho «una profunda revisión de las doctrinas económicas con la mira de encontrar los principios de una economía científica», de la que habían surgido, a su vez, dos grandes corrientes: la «escuela matemática» (Walras, Pareto, Pantaleoni), que pretendía una «completa exactitud en el cálculo económico», siendo el gran apoyo del dirigismo económico; y la «escuela vienesa», representada por Böhm Bawerk, Wieser y, más recientemente, Mises y Hayek, «una corriente rigurosamente científica», con cuyas ideas se proponía él desarrollar sus Lecciones.

Aunque Ballvé no se prodigó en citas, dada la concisión con que se propuso exponer los fundamentos de la economía, no faltan en sus Lecciones las de Mises, Wieser, Böhm Bawerk y Hayek.

1912, en la que habían venido conviviendo desde entonces diversos grupos de liberales mexicanos. Agustín Navarro sería Secretario del PAN y director del Instituto Ludwig von Mises de México. 
Y junto a ellas, aparecen también las de algunos de los economistas de la Mont Pelèrin y otros de la escuela austriaca, como Eucken, Röepke, Rueff o el propio Hazlitt, quien para entonces ya había publicado su Economics in one lesson (1946).

Respecto a este último, tiene especial interés preguntarse si Ballvé pudo haberse inspirado particularmente en su libro al escribir sus Lecciones, dado que ambos textos vienen citándose comúnmente como parte esencial del tronco misiano, como veremos más adelante. Por supuesto, lo había leído, porque lo cita en la Lección I para afirmar expresamente que Hazlitt coincidía con las ideas de Mises y Hayek. Sin embargo, el propósito de éste al publicar su Economics in one lesson y, por tanto, sus resultados, fueron muy distintos a los de Ballvé y sus Lecciones. Henry Hazlitt, un prestigioso periodista especializado en temas económicos, que escribía en el Wall Street Journal y otros grandes diarios americanos y que publicaba libros de divulgación de filosofía, política y economía, ${ }^{29}$ no se propuso realmente escribir un manual, sino tan sólo ofrecer «un análisis de los sofismas económicos que han alcanzado en los últimos tiempos preponderancia suficiente hasta convertirse casi en una nueva ortodoxia», haciéndolo a un nivel poco más que periodístico y utilizando fundamentalmente a Bastiat, Wicksteed y Mises, ${ }^{30}$ cuyo Human Action, no publicado aun, debía conocer bien por su asistencia habitual a los seminarios de la NYU. ${ }^{31}$ Las Lecciones de Ballvé, en cambio,

${ }^{29}$ Existe una edición española, traducida por Joaquín Reig Albiol, con el título de La ciencia de la economía en una sola lección (Valencia: Fundación Ignacio Villalonga. Biblioteca de Estudios Económicos, 1958). Otros libros de Hazlitt fueron publicados también en España en los años siguientes: La ciencia económica ante la «inutilidad» del socialismo (Valencia: Fundación Ignacio Villalonga. Biblioteca de Estudios Económicos, 1959), con textos de Eastman, Mises y Chamberlin; y Los errores de la nueva ciencia económica (Madrid: Aguilar, 1961).

30 Es interesante destacar cómo todavía en 1946, Hazlitt, uno de los escritores que más han contribuido a la difusión popular de la escuela austriaca, incluyera como fuentes comunes a corrientes como la escuela economista de Bastiat; el Wicksteed del The Common Sense of Polítical Economy (1910), que tras abandonar sus veleidades fabianas y georgistas se había convertido en el principal difusor de las ideas de Jevons; y el Mises de la NYU y de Human Action.

31 Pese a haber sido "compuesto de modo unitario», según su autor, tres de los veinticuatro capítulos habían sido publicados previamente como artículos en The New York Times y otros periódicos norteamericanos. 
sí fueron concebidas como un verdadero manual de Economía, y a un nivel analítico mucho más alto.

En cuando a su estructura formal, no es difícil observar cierta similitud entre las Lecciones de Ballvé y Human Action, aun cuando quede un tanto oscurecida, debido a que el economista catalán, al proponerse escribir un compendio de Economía, tuvo que prescindir de buena parte de las cuestiones filosóficas planteadas por Mises y que agrupar drásticamente los 39 capítulos de su tratado. Pese a ello, el iter común de ambos libros puede seguirse facilmente: i) concepto y método de la economía, con especial énfasis en la naturaleza de la acción humana y en las categorías de tiempo e incertidumbre; ii) mercados, precios y soberanía del consumidor; iii), empresa; iv) capital y trabajo; v) moneda y crédito; vi) explicación de las crisis económicas con los principios de la escuela austriaca, y crítica del desempleo de Marx y de la economía keynesiana; vii) comercio internacional; viii) nacionalismo y socialismo, dos desviaciones sin fundamentación analítica; ix) dirigismo económico, la más perniciosa y peligrosa de las intervenciones públicas; $\mathrm{x}$ ) y un capítulo final, muy breve, que tal vez añadió Ballvé para suplir en parte la ausencia de la introducción enviada a Sennholz, pensada y escrita seguramente para formar parte de las Lecciones, dedicado a cuestiones varias, como el «homo oeconomicus», el equilibriodesequilibrio económico y la justicia distributiva.

Al igual que Mises en su tratado y al contrario que Samuelson en su Economics: An Introductory Análisis (1945), uno de los primeros manuales de economía postkeynesianos, Ballvé integró plenamente la micro y la macroeconomía gracias a su teoría subjetiva del valor y a su explicación de las crisis económicas. ${ }^{32}$ Y, por otra parte, rechazó la habitual división de los libros de texto clásicos en producción, distribución y consumo, ${ }^{33}$ a las que él veía

32 Una explicación de la integración de la micro y la macro en la economía misiana, en J. Huerta de Soto, «Estudio Preliminar», en Mises, La acción humana (Madrid: Unión Editorial, 9. a edición, 2009: XXXVI-XXXVI).

33 Sobre la estructura de los manuales de economía clásicos, vid. M. Martín Rodríguez, «La institucionalización de los estudios de Economía Política en la Universidad española (1784-1857)», estudio introductorio a marqués de Valle Santero, 
como manifestaciones simultáneas de acciones volitivas de los individuos.

Nada esencial del sistema misiano falta en los contenidos de las Lecciones: i) la economía es una praxeología cataláctica, cuyas categorías esenciales son la elección humana, el intercambio de bienes y el mercado, en un tiempo y espacio determinados; ii) el empresario y la propiedad privada constituyen las únicas instituciones capaces de satisfacer las necesidades de los consumidores de un modo eficiente y, por tanto, el cálculo económico resulta totalmente imposible en las economías socialistas; iii) el intervencionismo económico lleva muy a menudo a resultados diametralmente opuestos a los buscados, y el dirigismo económico, indefectiblemente al socialismo; iv) el comercio internacional no presenta ninguna característica sustantiva que le haga distinguirse del comercio interior, y el proteccionismo y el nacionalismo económicos constituyen una aberración basada en falsas premisas y en realidades históricas muy concretas; v) el incremento de la circulación fiduciaria o la expansión crediticia en sistemas bancarios de reservas fraccionarias conducen inexorablemente a procesos inflacionarios que empobrecen a la comunidad $\mathrm{y}$, muy particularmente, a los más desfavorecidos; vi) estos procesos inflacionarios terminan, antes o después, en crisis o recesiones económicas en las que se ponen de manifiesto los errores cometidos en las decisiones de inversión, con la secuela de un paro masivo hasta que se liquidan los activos erróneamente producidos; vii) el precio del trabajo, como el de todos los factores de producción, se determina en el mercado y cualquier intento de alejarlo artificialmente de él se salda necesariamente con paro o con asignaciones ineficientes; y viii) la esencia de la economía no es el equilibrio, imposible de conseguir y que en todo caso conduciría al estancamiento, sino el desequilibrio, que constituye el motor del crecimiento, y de ahí que el problema económico fundamental consista en el estudio de los procesos dinámicos de coordinación social.

Elementos de Economía Política con aplicación particular a España, Madrid: Instituto de Estudios Fiscales, 1989 
Tan sólo cabe añadir que Ballvé tuvo que enfrentarse a un problema que Mises no se había siquiera planteado. Formado en la economía fabiana, el economista catalán había ido evolucionando hacia el liberalismo para terminar conociendo muy de cerca el colectivismo y el anarquismo. Para refutar el colectivismo disponía de los instrumentos analíticos misianos, pero el anarcocomunismo libertario había sido un movimiento propio de los países latinos y sólo había sido experimentado con todas sus consecuencias durante la guerra civil española, en la que si bien había sido impulsado inicialmente por un individualismo extremo, muy pronto terminó en un simple corporativismo, que exigió crecientes controles públicos y sindicales, con una negación absoluta del mercado. Ballvé, después de haber vivido esta experiencia, estaba en condiciones inmejorables para no confundir individualismo anarcocomunista libertario con libertad económica y lo hizo con todo rigor a todo lo largo de sus Lecciones.

Por otra parte, Ballvé demostró conocer bien la escuela clásica, el marxismo, la escuela neoclásica y el keynesianismo. Para contraponer a cada uno de estos paradigmas los principios de sus propias Lecciones, expuso con rigor y brillantez los fundamentos de la ciencia económica, siempre de forma breve y concisa y recurriendo a menudo a la historia económica y a la discusión de sofismas económicos en forma parecida a como lo había hecho Bastiat un siglo antes. Y para facilitar la lectura de su texto, puso generalmente en cursiva las definiciones y frases que quería destacar.

Finalmente, comoquiera que no pretendemos aquí hacer una exposición de cada uno de los capítulos de las Lecciones, llamaremos tan sólo la atención sobre otra de sus principales características: su crítica a las políticas económicas que se venían haciendo en los países latinoamericanos, que podemos analizar mediante tres casos distintos, muy significativos.

En esos años, México estaba viviendo los efectos perniciosos de la política inflacionista que se había venido practicando desde 1940 con el fin de promover la industrialización del país. Según dijimos más arriba, el soporte analítico para este tipo de políticas lo había dado, entre otros, W.A. Lewis, contra el que Ballvé arremetió de nuevo en sus Lecciones, ahora de forma más extensa y 
precisa. De acuerdo con su propio análisis, la política de dinero abundante y barato, piedra angular de estas políticas, conducía inexorablemente a precios y salarios altos y provocaba un auge ficticio que, al terminarse la inyección monetaria, ponía en evidencia los proyectos mal concebidos, haciendo que la producción de bienes inútiles condujese a situaciones de crisis, que no eran sino «el resultado de apartarse de la economía y sustituirla por la política» (1956b: 57).

De las nacionalizaciones que se habían venido haciendo en distintos países latinoamericanos, criticó en particular las que se habían apoyado en el concepto ricardiano de renta de la tierra, que había llevado, a través de Henry George y otras derivaciones, a las reformas agrarias de México y otros países. Ballvé criticó dos puntos fundamentales: de un lado, negó que hubiese escasez de tierras, ya que existían todavía muchas sin cultivar en el mundo y todo lo que había que hacer era permitir que la población se trasladase a cultivarlas; de otro, siguiendo a Mises, sostuvo que no había simplemente tierra sino tierras de distinta calidad que, como el resto de máquinas y utensilios, se pagaban a distintos precios, sin que por ello se pudiera decir que «usurpa una renta el que tiene una tierra de buena calidad cuya renta ya ha capitalizado al pagarla a un precio más alto» (1956b: 83).

Su crítica a los «dirigistas» o "planeadores» fue más dura. Decían éstos querer salvar la economía libre, pero de hecho no eran para Ballvé sino las «celestinas» del comunismo, como había expuesto Hayek en su Camino de servidumbre. Su propósito era corregir las debilidades del mercado, que «consisten en la falta de movilidad de los recursos, la injusta distribución de la riqueza y la incapacidad para hacer frente al comercio internacional» (1956b: 86), pero sus remedios eran precisamente los impuestos y subsidios, el control de precios y salarios, la intervención en la moneda y el control del comercio internacional, o sea, menos movilidad y menos producción. Y, además de todo ello, la intervención imponía a los gobiernos de países atrasados unas obligaciones de competencia y honestidad difíciles de encontrar en ellos. 


\section{La recepción de las «Lecciones»}

Las Lecciones de Ballvé (1956b), cuya primera edición, del Instituto de Investigaciones Sociales y Económicas de México, fue de 10.000 copias, pronto conocieron dos nuevas ediciones de este mismo Instituto, ambas de 1961, de 5.000 copias cada una. Parece evidente, pues, que no fueron sólo los alumnos de economía del ITAM y de otros centros universitarios sus únicos destinatarios, sino que debieron llegar a otras muchas manos en distintos países de Latinoamérica, distribuidas por los centros de inspiración liberal que se estaban creando en casi todos ellos por esos mismos años.

En 1957, se publicaron en la editorial Sedif (París), traducidas al francés por Raoul Audouin y con un prólogo de Pierre Lhoste-Lachaume, dos de los grandes pensadores del liberalismo francés del siglo XX. ${ }^{34}$ Con la expresa autorización de Ballvé en una nota de presentación que hizo al libro, el prologista cambió su título original por el de L'Economie vivante: son histoire et ses mecanismes e introdujo ciertas modificaciones en el texto, algunas de cierta importancia, para «adaptarlo» a los intereses del público francés y para poner de relieve determinados puntos a la luz de la situación europea.

En 1960, el Centro de Estudios Económico-Sociales (CEES) de Guatemala, fundado a finales de 1959, publicó la primera edición en español fuera de México, con el título de Fundamentos de la Ciencia Económica y un breve prólogo de Manuel F. Ayau en defensa de la economía de mercado, y con una tirada de 2.000 ejemplares. ${ }^{35}$ El mismo CEES publicaría dos nuevas ediciones en 1967

34 Pierre Lhoste Lachaume fue el fundador del Centre Libéral Spiritualiste Française (CLSF), al que llevó a un joven Raoul Audouin, que le sucedería en la presidencia del centro en 1973. Ambos fueron miembros de la Mont Pèlerin Society, en cuyas reuniones debió conocerlos Ballvé. Audouin, que en 1945 había traducido ya algunos extractos de The Road to Serfdom, de Hayek, también para la editorial Sedif, traduciría en los años siguientes los principales textos del liberalismo clásico y de la escuela austriaca: Mises, Hayek, Harod Berman, Irving Kristol, Israel Kirzner y Michael Novak, entre otros.

35 El Centro de Estudios Económico-Sociales (CEES) de Guatemala fue fundado en 1958, a imagen del Instituto mexicano del mismo nombre, por Manuel Francisco 
y 1984, con una tirada de 6.000 y 2.000 ejemplares, respectivamente. ${ }^{36}$

En 1963, apareció la primera edición en inglés, en la editorial D. Van Nostrand Company, Inc. (Princeton, Toronto, London, New York), con el título de Essential of Economics: A brief Survey of Principles and Policies, con traducción y prólogo de Arthur Goddard y una brevísima presentación de Felix Morley. ${ }^{37}$ La traducción de Goddard, pese a haberla hecho directamente del español, tuvo en cuenta algunos de los cambios introducidos por Lhoste-Lauchame en la edición francesa, cuando, según el propio traductor, «it seemed to represent an improvement, in vigor and consistency of expresión, over de Spanish original». ${ }^{38}$

La misma traducción de Goddard, y el mismo título, sirvieron a The Foundation for Economic Education (FEE) para hacer sus tres ediciones sucesivas de 1969, 1994 y $1997 .{ }^{39}$ En la nota de

Ayau Cordón y un grupo de empresarios guatematecos, con el objetivo de «estudiar y difundir los principios éticos, económicos y jurídicos de la sociedad libre». Ayau (1925-2010), ingeniero y doctor en Derecho en universidades norteamericanas, estuvo toda su vida al frente de un importante grupo empresarial y fundó y fue presidente de la Bolsa de Valores de Guatemala. Fue, asimismo, miembro de la Mont Pelèrin Society, de la que fue presidente durante 1978-80; fiduciario de la Foundation for Economic Education de Nueva York; y miembro de la Junta Directiva del Liberty Fund de Indianápolis. También fue un destacado político liberal, miembro de la Asamblea Legislativa y candidato a la presidencia de Guatemala. En 2008, recibió el premio Juan de Mariana del Instituto Juan de Mariana de España. Del CEES surgió en 1971 la prestigiosa Universidad Francisco Marroquín, de la que Ayau fue su primer rector.

36 Alguna referencia apunta a una temprana edición de las Lecciones en Buenos Aires, pero no he podido localizarla en ninguna de las bibliotecas públicas consultadas.

37 Arthur Goddard, muy vinculado a Mises, con quien publicó algunos trabajos, tradujo al inglés otros textos de economía relacionados con el liberalismo, como los Sofismas Económicos de Bastiat. Felix Morley fue muchos años editor del The Washington Post, premio Pulitzer y periodista de la NBC, en la que adquirió un gran prestigio, sobre todo después de la publicación de Freedom and Federalism, compendio de sus ideas políticas y económicas en defensa del liberalismo.

${ }^{38}$ F. Ballvé (1963): Essentials of Economics, Princeton, Toronto, London, New York: D. Van Nostrand Company, Inc, p. ix.

${ }^{39}$ F. Ballvé (1997), Essentials of Economics: New York, The Foundation for Economic Education, Inc., página v. La FEE, con sede en New York, había sido fundada en 1946 por Leonard Read, gerente de la Cámara de Comercio de Los Ángeles, como una institución sin fines de lucro para promover el libre mercado y las ideas de libertad, 
presentación del editor se decía: «The author has succeeded in presenting the essential teachings of economics in an eminently clear and readable form ideally suited to the needs of the citizen who seeks to acquaint himself with the knowledge necessary for a wel-founded and rationally defendible opinión concerning the consequences of proposed policies that are courrently the subjet of controversy. Illustrated with apt historical references and buttressed with solid learning, this work is a rare combination of sound scholarship an pedagogic skill».

La última edición en inglés ha sido la del Ludwig von Mises Institute (Auburn, Alabama, USA), de 2008, que incluye los prefacios en español y en inglés y el prólogo de Morley. ${ }^{40}$

Después de esta relación de las sucesivas ediciones de las Lecciones, veamos cual fue su recepción. La primera reseña que se publicó sobre ellas fue la de Agustín Navarro, uno de los fundadores del Instituto de Investigaciones Sociales y Económicas A.C., aparecida en la Revista de la Facultad de Derecho de la UNAM (n. -23 , julio-septiembre 1956). En ella se calificaba ya al economista catalán como «el representante más destacado del liberalismo y de la escuela austriaca en México", aunque añadiendo que «el autor tiene demasiada personalidad, demasiado sentido universal, demasiado vitalismo para caber dentro de cualquier ortodoxia». En el mismo número de la revista, el propio Ballvé reseñaba un libro de Miguel Mancera Aguayo, Aspectos de las relaciones entre las inversiones internacionales, el ingreso nacional y el movimiento de divisas en un país subdesarrollado, su tesis doctoral en el ITAM, en el que se defendían las inversiones internacionales como vía para el desarrollo económico.

mediante la publicación de libros y folletos, el patrocinio de seminarios y conferencias y la reimpresión de textos liberales clásicos. En 1947 ayudó a Hayek a la creación de la Mont Pelèrin Society. Entre sus presidentes, ha estado el prestigioso economista de la escuela austriaca Richard M. Ebeling (2003-2008).

40 El Ludwig von Mises Institute fue fundado en 1982 por Lewellyn Rockwell Jr., con la aprobación de Margit von Mises, como un centro de investigación y enseñanza, con una clara orientación hacia el liberalismo clásico y la escuela austriaca de economía. Sus primeros presidentes fueron la propia Margit von Mises, Hayek, Fertig, Hazlitt y Rothbard. Entre sus publicaciones periódicas está The Quaterly Journal of Austrian Economics. 
Como cabía esperar, la primera edición en inglés de las Lecciones no fue bien recibida en determinados medios académicos. Baste como ejemplo la reseña publicada en Political Research Quaterly (1963: 985-986), cuyo autor no estaba dispuesto a admitir los fundamentos de la economía austriaca, pese a que en ningún momento vinculara expresamente a Ballvé con esta escuela. Siguiendo en su crítica el guión convencional de los manuales de economía que se estudiaban por entonces en las universidades de casi todo el mundo, admitía que la primera parte del libro, relativa a las cuestiones consideradas de microeconomía, ofrecía una «reasonably lucid and accurate presentation of the interaraction of supply and demand», pero la segunda, en la que se discutían los agregados económicos, el dinero, la política monetaria y el comercio internacional, contenía, en cambio, «many misrepresentations and misstatements as to leave the reader with a false and confused picture». En particular, el capítulo 5, sobre el dinero y el crédito, era el que le parecía menos aceptable de todo el libro, acusando a su autor de no haber tenido en cuenta las contribuciones hechas a la ciencia económica en los últimos cincuenta años y de no entender el papel del gobierno en una «sociedad democrática».

En pleno auge del keynesianismo y de la síntesis neoclásica, era normal que las Lecciones ni tan siquiera se reseñaran con referencia a la economía austriaca, prácticamente ignorada en esos años. Sin embargo, a partir de su publicación en inglés por la FEE en 1969, Ballvé pasó a ser considerado como uno de los grandes economistas de la escuela austriaca y su manual como una introducción insuperable para cuantos quisieran aproximarse a esta escuela, habiendo ejercido desde entonces una notable influencia sobre toda una generación de economistas en centros académicos orientados hacia la economía liberal y austriaca. ${ }^{41}$ En una extensa reseña, John Chamberlain ${ }^{42}$ (The Freeman, 1969) dijo de

41 Basta para comprobarlo con visitar las páginas web de estos centros, algunos de ellos citados en notas anteriores.

42 John Chamberlain (1903-1995) fue un periodista americano, autor de varios libros sobre el capitalismo, muy influenciado por Albert Jay Nock y Ayn Rand. A partir de 1946, fue el editor de The Freeman, un magazine liberal fundado por Leonard Read, de la FEE, hasta el momento en que le sucedió Hazlitt. 
las Lecciones: «If you want instant enlightement, Henry Hazlitt's Economics in One Lesson is still the desired text. If you want enlightement in great depth, there Mises's Human Action. But if you are looking for something in the "between" category, Faustino Ballvé's Essentials of Economics: A brief Survey of Principles and Policies, traslated from the Spanish by Arthur Goddard, is your meat». Y el propio Hazlitt, en un artículo sobre los fundamentos de la economía austriaca (The Freeman, febrero 1981), al hacer una lista de lecturas, las recomendó expresamente «antes de que lector pasara» a The Fallacy of the Mixed Economy, de Stephen C. Licttlechild, también un librito de 85 páginas, y luego a los Principios de Menger y a La acción humana de Mises.

Con la reciente edición del Mises Institute de 2008, el interés por Ballvé ha aumentado extraordinariamente. Jeffrey A. Tucker, vicepresidente editorial del Mises Institute, profesor de la Acton University y autor de una bibliografía anotada de Hazlitt, orgulloso de haber redescubierto un tesoro, ha dicho de las Lecciones: "After you read Economics in One Lesson, this is an excellent book to take you to the next level. Hazlitt covers the general lesson and appplications, but this book zeros in on particular aspects of theory that are essential for stage two».

Queda, por último, conocer la suerte de las Lecciones en España. Como todos los economistas del exilio republicano, Ballvé ha sido olvidado durante muchos años. Joaquín Reig Albiol, ${ }^{43}$ un economista español de la escuela austriaca, también injustamente olvidado, se refirió a ellas muy tempranamente en dos ocasiones. La primera fue en una nota a pie de página en su prólogo a la edición española del libro de Hazlitt, La ciencia de la

43 Sobre Reig Albiol, vid. J. Huerta de Soto, «Ignacio Villalonga: Semblanza de un político, banquero y liberal», en Nuevos estudios de Economía Política, Madrid: Unión Editorial; y G. Tortella Casares (2000): «Ignacio Villalonga Villalba: 1895-1973», en Los 100 empresarios españoles del siglo XX, Madrid: Editorial Lid. Joaquín Reig, que había hecho su tesis doctoral sobre Mises, estuvo encargado durante años de traducir y editar, con estudios introductorios muy notables, los textos clásicos de economía liberal, principalmente de la escuela austriaca, que publicó la Fundación Ignacio Villalonga a partir de su creación en 1956. Durante estos años estuvo muy vinculado a otros centros liberales de todo el mundo y, en particular, a la Universidad Francisco Marroquín, de la que fue nombrado doctor honoris causa. 
economía en una sola lección, publicada en 1958, en la que Reig decía haber tomado algunas de las referencias históricas de las Lecciones para redactar su propio prólogo. Y la segunda, también en una nota a pie de página en su prólogo a la edición española de La acción humana, publicada por la Biblioteca de Estudios Económicos en 1960, en la que hacía una referencia a la impugnación por Ballvé del influyente libro de Lewis, La planeación económica. Después, con muy contadas excepciones, como la de $\operatorname{Roca}^{44}$ y, más recientemente, la de Martín Rodríguez y Fernández Clemente, ${ }^{45}$ un olvido prácticamente absoluto.

${ }^{44}$ Frances Roca (1996), destacando el éxito internacional de las Lecciones, reprodujo su capítulo 10, en versión inglesa, en El pensament econòmic català (1900-1970), II, La transmissió de les idees econòmiques, Barcelona: Publicacions de la Universitat de Barcelona, pp. 250-254. En esta misma antología se recoge un texto de Joaquín Reig Albiol, destacando su sintonía con los «neoliberales exiliados»: Ballvé en México, Prados Arrarte en Buenos Aires y Trias Fargas en Nueva York.

45 En Martín Rodríguez y Fernández Clemente (2009), se hace un esbozo biográfico y una exposición del contenido de las Lecciones y de la significación de Ballvé en el exilio republicano. 